\title{
A SHORT PROOF OF THE CUSHING-HENSON CONJECTURE
}

STEVO STEVIĆ

Received 8 June 2006; Revised 21 September 2006; Accepted 27 September 2006

We give a short proof of the Cushing-Henson conjecture concerning Beverton-Holt difference equation, which is important in theoretical ecology. The main result shows that a periodic environment is always deleterious for populations modeled by the BevertonHolt difference equation.

Copyright (C) 2006 Stevo Stević. This is an open access article distributed under the Creative Commons Attribution License, which permits unrestricted use, distribution, and reproduction in any medium, provided the original work is properly cited.

\section{Introduction}

Recently there has been a great interest in studying nonlinear difference equations and systems, in particular those which model some real life situations in population biology and ecology, see, for example, [1-13], and the references therein.

In [3], among other things, Cushing and Henson investigate the positive solutions of a special case of the Beverton-Holt difference equation [2]

$$
x_{n+1}=\frac{\mu K_{n} x_{n}}{K_{n}+(\mu-1) x_{n}}, \quad n \in \mathbb{N}_{0}
$$

where $\mu>1$ and $K_{n}=K_{n+p}, n \in \mathbb{N}_{0}$, for some $p \geq 2$.

Equation (1.1) with $K_{n}=K, n \in \mathbb{N}_{0}$, appeared in theoretical ecology, where the parameters $\mu$ and $K$ play an important role. The coefficient $\mu$ is considered a characteristic of the population (its intrinsic growth rate), determined by life cycle and demographic properties such as birth rates, survivorship rates, and so forth. The coefficient $K$ is considered a characteristic of the habitat or environment (called the carrying capacity), for example, resource availability, temperature, humidity, and so forth. It is quite common for biological populations that parameters fluctuate in time. For example, in the case of (1.1), periodic fluctuations are common (caused, e.g., by annual or daily fluctuations in the physical environment), in which case $\mu$ and/or $K$ become periodic functions of time. Hence, it is of interest to consider nonautonomous (1.1). 
In [4] Cushing and Henson posed the following two conjectures.

Conjecture 1.1. There is a positive $p$-periodic solution $\left\{\bar{x}_{0}, \ldots, \bar{x}_{p-1}\right\}$ of $(1.1)$ and it globally attracts all positive solutions.

Conjecture 1.2. The following inequality holds $(1 / p) \sum_{i=0}^{p-1} \bar{x}_{i}<(1 / p) \sum_{i=0}^{p-1} K_{i}$.

In a private communication with Professor Elaydi we found out that both conjectures have been confirmed recently by Elaydi and Sacker (the first one in [5] and the second one in [6]). Moreover, they have shown that (1.1) possesses a positive solution with period $p$, but it need not be the minimal period of the solution. Case $p=2$ has been solved in [3].

Here, we present our proofs of these two conjectures, which are simpler than those in the existing literature. The reason for the simplicity relies on the fact that (1.1) is solvable (as a special case of Riccati equation). The main result in this note, Theorem 1.3, confirms Conjecture 1.2, which is more interesting than Conjecture 1.1.

First note that by the change of variable $y_{n}=1 / x_{n},(1.1)$ can be written in the form

$$
y_{n+1}=q y_{n}+(1-q) L_{n}, \quad n \in \mathbb{N}_{0},
$$

where $q=1 / \mu$ and $L_{n}=1 / K_{n}$.

Since (1.1) and (1.2) are equivalent, it is enough to find $p$-periodic solutions of (1.2).

It is not difficult to find periodic solutions of (1.2), namely, if $\left(\bar{y}_{0}, \bar{y}_{1}, \ldots, \bar{y}_{p-1}\right)$ is such a solution, from (1.2), we have that it must be

$$
\bar{y}_{1}=q \bar{y}_{0}+(1-q) L_{0}, \quad \bar{y}_{2}=q \bar{y}_{1}+(1-q) L_{1}, \ldots, \bar{y}_{0}=q \bar{y}_{p-1}+(1-q) L_{p-1} .
$$

By iterating, or by solving linear system (1.3), we obtain

$$
\bar{y}_{0}=\frac{(1-q)\left[L_{0} q^{p-1}+\cdots+L_{p-2} q+L_{p-1}\right]}{1-q^{p}}
$$

and by cyclicity of system (1.3), it follows that

$$
\bar{y}_{i}=\frac{(1-q)\left[L_{\sigma^{[i]}(0)} q^{p-1}+\cdots+L_{\sigma^{[i]}(p-2)} q+L_{\sigma^{[i]}(p-1)}\right]}{1-q^{p}}, \quad i \in\{1, \ldots, p-1\},
$$

where $\sigma$ is the permutation $(0,1,2, \ldots, p-1) \rightarrow(1,2, \ldots, p-1,0), \sigma^{[i]}=\sigma \circ \sigma^{[i-1]}$ and $\sigma^{[0]}=$ Id (the identity permutation).

Hence,

$$
\bar{x}_{i}=\frac{\mu^{p}-1}{(\mu-1) \sum_{j=0}^{p-1}\left(\mu^{j} / K_{\sigma^{[i]}(j)}\right)}=\frac{1}{\sum_{j=0}^{p-1}\left(\alpha_{j} / K_{\sigma^{[i]}(j)}\right)},
$$

for $i \in\{0,1, \ldots, p-1\}$, where

$$
\alpha_{j}=\frac{(\mu-1) \mu^{j}}{\mu^{p}-1}, \quad j \in\{0,1, \ldots, p-1\}
$$

Now we are in a position to confirm Conjecture 1.2 in an elegant way by proving the following result. 
Theorem 1.3. Consider (1.1), where $\mu>1$ and $K_{n}$ is a nonconstant sequence of positive numbers such that $K_{n}=K_{n+p}, n \in \mathbb{N}_{0}$, for some $p \geq 2$. If $\left\{\bar{x}_{0}, \ldots, \bar{x}_{p-1}\right\}$ is the positive $p$ periodic solution of (1.1), then

$$
\frac{1}{p} \sum_{i=0}^{p-1} \bar{x}_{i}<\frac{1}{p} \sum_{i=0}^{p-1} K_{i}
$$

Proof. Since the function $f(x)=1 / x$ is strictly convex on $(0, \infty)$ and $\sum_{j=0}^{p-1} \alpha_{j}=1$, we have

$$
\sum_{i=0}^{p-1} \bar{x}_{i}=\sum_{i=0}^{p-1} \frac{1}{\sum_{j=0}^{p-1}\left(\alpha_{j} / K_{\sigma^{[i]}(j)}\right)}<\sum_{i=0}^{p-1} \sum_{j=0}^{p-1} \alpha_{j} K_{\sigma^{[i]}(j)}=\sum_{j=0}^{p-1} \alpha_{j} \sum_{i=0}^{p-1} K_{\sigma^{[i]}(j)}=\sum_{i=0}^{p-1} K_{i}
$$

where the inequality is strict since the sequence $K_{n}$ is nonconstant.

The interpretation of Theorem 1.3 is that a periodic environment is always deleterious for populations modelled by (1.1) in the sense that the average of the resulting population oscillations is strictly less than the average of $K$ 's.

Remark 1.4. Conjecture 1.1 can be confirmed in a similar fashion. Namely, similar to (1.4), for arbitrary solution $\left(y_{n}\right)_{n \in \mathbb{N}_{0}}$ of (1.1) the following relationship holds:

$$
y_{m p+i}=q^{p} y_{(m-1) p+i}+(1-q)\left[L_{\sigma^{[i]}(0)} q^{p-1}+\cdots+L_{\sigma^{[i]}(p-2)} q+L_{\sigma^{[i]}(p-1)}\right],
$$

$m \in \mathbb{N}_{0}$ and $i \in\{0,1, \ldots, p-1\}$, and consequently

$$
y_{m p+i}=q^{m p} y_{i}+\frac{1-q^{m p}}{1-q^{p}}(1-q)\left[L_{\sigma^{[i]}(0)} q^{p-1}+\cdots+L_{\sigma^{[i]}(p-2)} q+L_{\sigma^{[i]}(p-1)}\right] \text {, }
$$

$m \in \mathbb{N}_{0}$ and $i \in\{0,1, \ldots, p-1\}$.

Letting $m \rightarrow \infty$ in the last formula for each $i \in\{0,1, \ldots, p-1\}$, and noticing that $q \in$ $(0,1)$, we obtain that every solution of $(1.2)$ converges to the $p$-cycle $\left(\bar{y}_{0}, \ldots, \bar{y}_{p-1}\right)$, which implies that every solution of (1.1) converges to the $p$-cycle $\left(\bar{x}_{0}, \ldots, \bar{x}_{p-1}\right)$, as desired.

Remark 1.5. One of the referees of this paper noted that the existence of a periodic solution of (1.1), as well as its global attractivity, was proved in [10]. However, in [10] the periodic solution was not found in a closed form and Conjecture 1.2 was not considered. Actually, the problem in Conjecture 1.1 is folklore and it is difficult to find its origins.

Remark 1.6. If $\mu$ in (1.1) is not constant but periodic with period $p$, for example, $\mu_{n}=$ $\mu_{n+p}$, then Conjecture 1.1 is also true. Indeed, in this case (1.2) becomes

$$
y_{n+1}=q_{n} y_{n}+\left(1-q_{n}\right) L_{n}, \quad n \in \mathbb{N}_{0},
$$

where $q_{n}=1 / \mu_{n}$. As above it can be obtained that

$$
\bar{y}_{i}=\frac{\sum_{j=0}^{p-1}\left(1-q_{\sigma^{[i]}(j)}\right) L_{\sigma^{[i]}(j)} \prod_{k=j+1}^{p-1} q_{\sigma^{[i]}(k)}}{1-\prod_{j=0}^{p-1} q_{j}}, \quad i \in\{0,1, \ldots, p-1\},
$$


and that arbitrary solution $\left(y_{n}\right)_{n \in \mathbb{N}_{0}}$ of the equation has the form

$$
y_{m p+i}=\left(\prod_{j=0}^{p-1} q_{j}\right)^{m} y_{i}+\frac{1-\left(\prod_{j=0}^{p-1} q_{j}\right)^{m}}{1-\prod_{j=0}^{p-1} q_{j}} \sum_{j=0}^{p-1}\left(1-q_{\sigma^{[i]}(j)}\right) L_{\sigma^{[i]}(j)} \prod_{k=j+1}^{p-1} q_{\sigma^{[i]}(k)},
$$

$m \in \mathbb{N}_{0}$ and $i \in\{0,1, \ldots, p-1\}$.

Letting $m \rightarrow \infty$ in (1.14) it follows that $y_{m p+i} \rightarrow \bar{y}_{i}$, for each $i \in\{0,1, \ldots, p-1\}$, from which the result follows.

From (1.13), we have that

$$
\bar{x}_{i}=\frac{\prod_{k=0}^{p-1} \mu_{k}-1}{\sum_{j=0}^{p-1}\left(\left(\mu_{\sigma^{[i]}(j)}-1\right) / K_{\sigma^{[i]}(j)}\right) \prod_{k=0}^{j-1} \mu_{\sigma^{[i]}(k)}}, \quad i \in\{0,1, \ldots, p-1\} .
$$

Let

$$
\alpha_{i, j}=\frac{\left(\mu_{\sigma^{[i]}(j)}-1\right) \prod_{k=0}^{j-1} \mu_{\sigma^{[i]}(k)}}{\prod_{k=0}^{p-1} \mu_{k}-1}, \quad i, j \in\{0,1, \ldots, p-1\} .
$$

Then, similar to the proof of Theorem 1.3, we obtain

$$
\begin{aligned}
\sum_{i=0}^{p-1} \bar{x}_{i} & =\sum_{i=0}^{p-1} \frac{1}{\sum_{j=0}^{p-1}\left(\alpha_{i, j} / K_{\sigma^{[i]}(j)}\right)}<\sum_{i=0}^{p-1} \sum_{j=0}^{p-1} \alpha_{i, j} K_{\sigma^{[i]}(j)} \\
& =\sum_{j=0}^{p-1} K_{j} \sum_{i=0}^{p-1} \alpha_{i, j-i(\bmod p)} \\
& =\sum_{j=0}^{p-1} K_{j} \frac{\left(\mu_{j}-1\right) \sum_{i=0}^{p-1} \prod_{k=0}^{j-i-1(\bmod p)} \mu_{\sigma^{[i]}(k)}}{\prod_{k=0}^{p-1} \mu_{k}-1} .
\end{aligned}
$$

From (1.17) it follows that

$$
\frac{1}{p} \sum_{i=0}^{p-1} \bar{x}_{i}<\frac{\left(\max _{i} \mu_{i}\right)\left(\max _{i} \mu_{i}-1\right)}{\left(\min _{i} \mu_{i}\right)\left(\min _{i} \mu_{i}-1\right)} \frac{1}{p} \sum_{i=0}^{p-1} K_{i}
$$

which gives an estimate of the average $(1 / p) \sum_{i=0}^{p-1} \bar{x}_{i}$ for the case when $\mu_{n}$ is a nonconstant periodic sequence, see [7].

\section{References}

[1] L. Berg and L. von Wolfersdorf, On a class of generalized autoconvolution equations of the third kind, Zeitschrift für Analysis und ihre Anwendungen 24 (2005), no. 2, 217-250.

[2] R. J. Beverton and S. J. Holt, On the Dynamics of Exploited Fish Populations, Fish. Invest., vol. 19, HMSO, London, 1957.

[3] J. M. Cushing and S. M. Henson, Global dynamics of some periodically forced, monotone difference equations, Journal of Difference Equations and Applications 7 (2001), no. 6, 859-872.

[4] _ A periodically forced Beverton-Holt equation, Journal of Difference Equations and Applications 8 (2002), no. 12, 1119-1120. 
[5] S. Elaydi and R. J. Sacker, Global stability of periodic orbits of non-autonomous difference equations and population biology, Journal of Differential Equations 208 (2005), no. 1, 258-273.

[6] Gl_ Global stability of periodic orbits of nonautonomous difference equations in population biology and the Cushing-Henson conjectures, Proceedings of the 8th International Conference on Difference Equations and Applications, Chapman \& Hall/CRC, Florida, 2005, pp. 113-126.

[7] Nonautonomous Beverton-Holt equations and the Cushing-Henson conjectures, Journal of Difference Equations and Applications 11 (2005), no. 4-5, 337-346.

[8] H.-F. Huo and W.-T. Li, Permanence and global stability of positive solutions of a nonautonomous discrete ratio-dependent predator-prey model, Discrete Dynamics in Nature and Society 2005 (2005), no. 2, 135-144.

[9] M. R. S. Kulenović and G. Ladas, Dynamics of Second Order Rational Difference Equations. With Open Problems and Conjectures, Chapman \& Hall/CRC, Florida, 2002.

[10] P. Liu and K. Gopalsamy, On a model of competition in periodic environments, Applied Mathematics and Computation 82 (1997), no. 2-3, 207-238.

[11] S.-E. Takahasi, Y. Miura, and T. Miura, On convergence of a recursive sequence $x_{n+1}=f\left(x_{n-1}, x_{n}\right)$, Taiwanese Journal of Mathematics 10 (2006), no. 3, 631-638.

[12] L.-L. Wang and W.-T. Li, Existence and global attractivity of positive periodic solution for an impulsive delay population model, Dynamics of Continuous, Discrete \& Impulsive Systems. Series A. Mathematical Analysis 12 (2005), no. 3-4, 457-468.

[13] X.-X. Yan, W.-T. Li, and Z. Zhao, On the recursive sequence $x_{n+1}=\alpha-\left(x_{n} / x_{n-1}\right)$, Journal of Applied Mathematics \& Computing 17 (2005), no. 1-2, 269-282.

Stevo Stević: Mathematical Institute of the Serbian Academy of Science, Knez Mihailova 35/I, 11000 Beograd, Serbia

E-mail address: sstevic@ptt.yu 


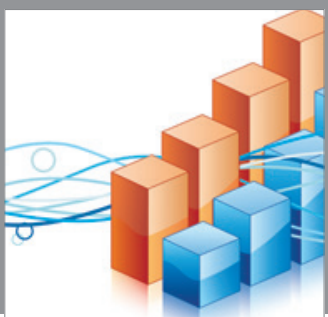

Advances in

Operations Research

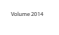

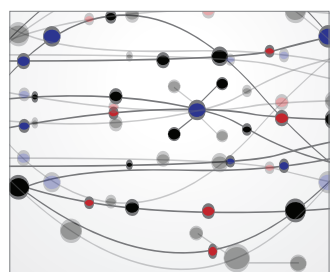

\section{The Scientific} World Journal
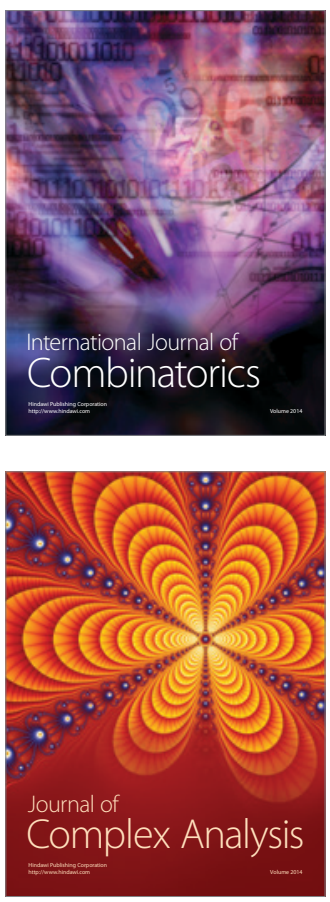

International Journal of

Mathematics and

Mathematical

Sciences
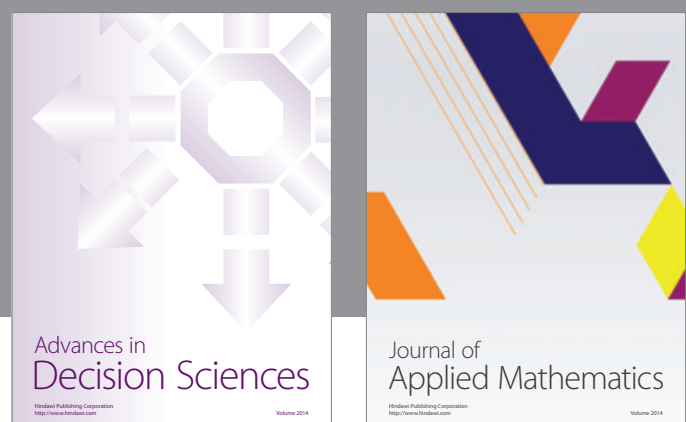

Journal of

Applied Mathematics
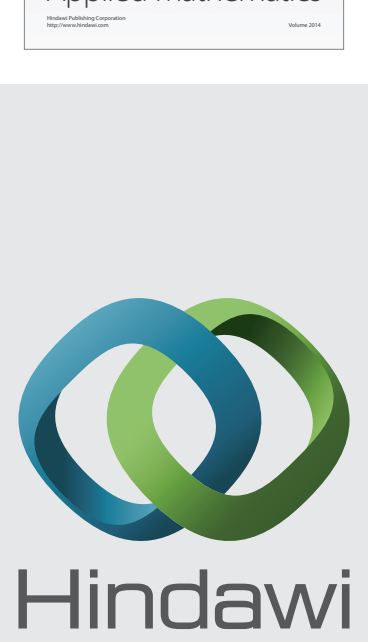

Submit your manuscripts at http://www.hindawi.com
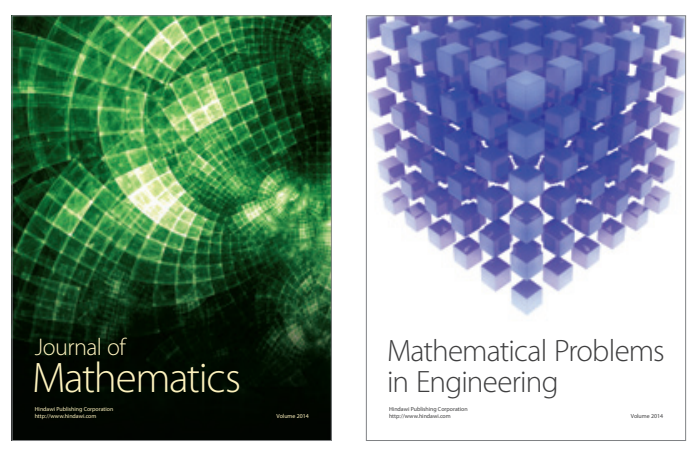

Mathematical Problems in Engineering
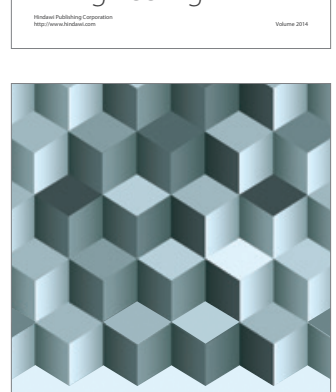

Journal of

Function Spaces
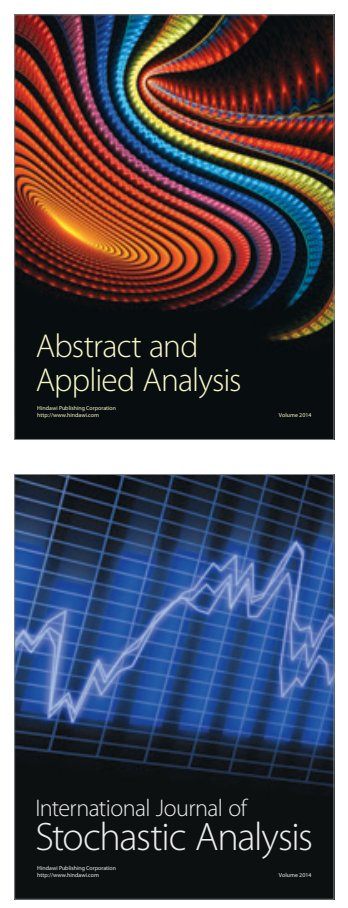

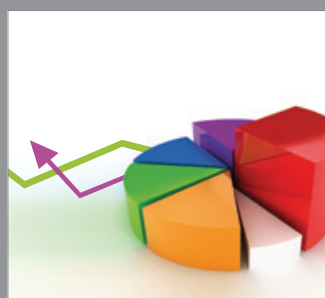

ournal of

Probability and Statistics

Promensencen
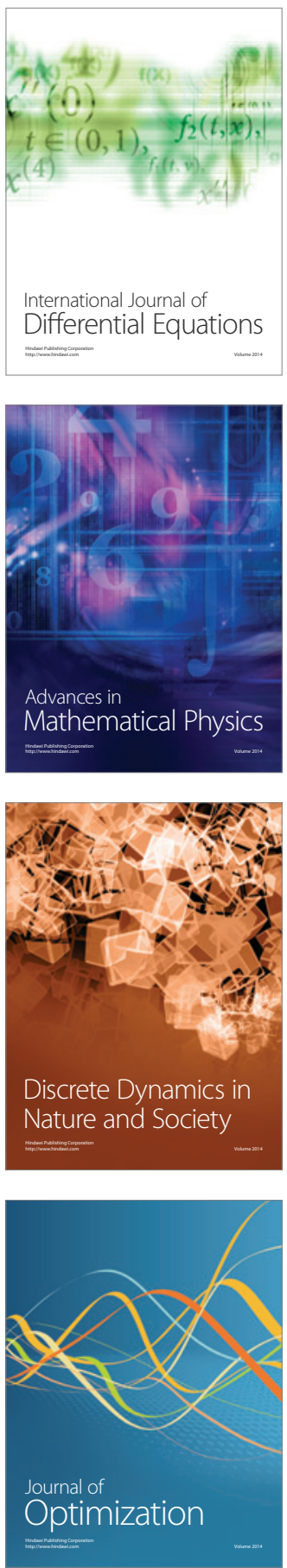